\title{
A Modified Fast Laser-Doppler Flowmetry to Measure the Pulsatile Microcirculation in Rats
}

\author{
Ming-Yie Jan ${ }^{1,2}$, Hsin. Hsiu ${ }^{1,2}$, Yuh-Ying Lin Wang ${ }^{3}$, Wei-Kung Wang ${ }^{1,2}$ \\ 1. Department of Electric Engineering, National Taiwan University, Taipei, Taiwan, R.O.C. \\ 2. Biophysic Lab., Institute of Physics, Academia Sinica, Taipei, Taiwan, R.O.C. \\ 3. Department of Physics, National Taiwan Normal University, Taipei, Taiwan, R.O.C.
}

\begin{abstract}
A fast Laser-Doppler flowmetery (fLDF) modified from a commercial one to measure the pulsatile microcirculation in rats. The time constant (TC) of the integrator in the analogy circuit of the LDF was modified; the frequency response of the modification was simulated by PSPICE to estimate the high $3 \mathrm{~dB}$ frequency. The coefficients of variance (CV) of 32 2 -secod ensembles of flux in the calibration standard measured by the commerical LDF and the modified one were less than $2 \%$. The animal tests also clearly demonstrated the pulsatile microcirculation on the renal cortex in the rat.
\end{abstract}

Key Words: LDF, Pulsatile Microcirculation, Time Constant

\section{Introduction}

The pulsatile flow in microcirculation has been debated for two decades, since the marcohemodynamic point of view assumed that the pulsatility is totally damped. Meanwhile, some distinguish scientists observed the pulsation of microcirculation indirectly by the dual-slit photometric technique in cat omentum [1] and directly by an intravital microscopy system in frog skeletal muscle [2] and in goat skeletal muscle [3]. Due to the illumination and tissue preparation, the pulsatility in a solid organ has not explored by the above methods.

A much more convenient tool, Laser-Doppler flowmetery (LDF), which was frequently used in the study of microcirculation [4] in the last two decades provides a better way to exceed the limitation. According to the Bonner's model [5], LDF can observe the averaged behaviors, flux and velocity, of a group of red blood cells (RBCs) flow into numerously randomized capillaries and arterioles under the cubic zone of the probe. We had use a commercial LDF to explored the pulsatile flux on the renal cortex in rats [6]. Due to the tradeoff of the signal to noise ratio (SNR) and the high frequency response, the current commercial LDF can only resolve the frequency up to $20 \mathrm{~Hz}$. In a rat model, the heart rate is about 6 to $8 \mathrm{~Hz}$; LDF can only observe the frequency structure up to the 3 rd harmonic.

In this research, we modified the time constant (TC) in the integrator circuit of a commercial LDF as a fast LDF. The fast LDF satisfied the need to resolve the pulsatile flux up to the 6 th harmonics $(48 \mathrm{HZ})$. The coefficients of variance (CV) of 32 2-secod ensembles of flux in the calibration standard measured by the commerical LDF and the modified one were estimated; both were less than $2 \%$. A preliminary animal test was also demonstrated that the modified LDF can clearly observe the pulsatile microcirculation on the renal cortex in the rat.

\section{Methods and Materials}

\section{A. Modified $L D F$}

A commercial LDF, MBF3 (Moor Instruments Ltd, England), was used. The TC of the analogy output of MBF3 is about 0.05 second; MBF3 samples the analogy signal with $40 \mathrm{~Hz}$ sampling rate and then converts it into the analogy output by a D/A converter (DAC). In the modified fast LDF, the TC of the integrator in the analogy circuit was modified. The analogy output was directly connected to a 486PC with a sample \& hold card and an A/D board. The frequency responses of the integrators of both systems were simulated by PSPICE to estimate the high $3 \mathrm{~dB}$ frequency.

\section{B. Experimental Setup}

In the commercial one, the flux signals of MBF3 were recorded from the DAC output that has $40 \mathrm{~Hz}$ data rate. In the modified one, the flux signal was recorded from the analogy output of the integrator. Both were sent to a simultaneous sample \& hold card AX753 (AXIOM Technology Co., LTD. Taiwan, R.O.C.) and then were connected to a $486 \mathrm{PC}$ with A/D converter board AX5621 (AXIOM Technology) in $1500 \mathrm{~Hz}$ sampling rate.

\section{Calibration and Animal Test.}

An optical fibre probe (P10M+P17, plastic fiber $500 \mu \mathrm{m}$ O.D., Moor Instruments Ltd.) were connected to the LDF and then calibrated by the motility flux standard (Moor Instruments Ltd.). The standard contains $0.5 \% \quad 0.482 \mu \mathrm{m}$ uniform polystyrene spheres in purified water with $0.01 \%$ surfactant to reduce conglomeration. The micro-spheres move in the water like the Brownian motion. Using both system to measure the flux in the standard, we acquired 32 2-second ensembles and compared the coefficients of variance $(\mathrm{CV})$ between them.

In the animal tests, two wistar rats were used to compare the difference between the two systems. The rats were 
anesthetized with Urethane $(700 \mathrm{mg} / \mathrm{kg}$ ip) and were placed on a heated pad to keep the body temperature. The blood pressure pulses were recorded by cannulating the I.V. catheter (Becton-Dickinson, USA) from the caudal artery to the abdominal aorta of the rats with a catheter-tip pressure transducer (P10EZ, Viggo-Spectramed, USA). The left kidney was exposed from the dorsal side of the rat and fixed with the cotton balls around it. The fatty capsule was separated. The optical fibre probe was put gently on the surface of the renal cortex to avoid the movement artifact introduced by the respiration or other movements.

\section{Results}

From the PSPICE simulation, the high $3 \mathrm{~dB}$ frequency of MBF3 is at $23 \mathrm{~Hz}$; the one of the modified LDF is at $48 \mathrm{~Hz}$. Thus, the new system can resolve the flux signal in a rat model up to the 6 th harmonics. The flux measured in the calibration standard at $24^{\circ} \mathrm{C}$ using $\mathrm{MBF} 3$ was $92.0 \pm 1.4$ units

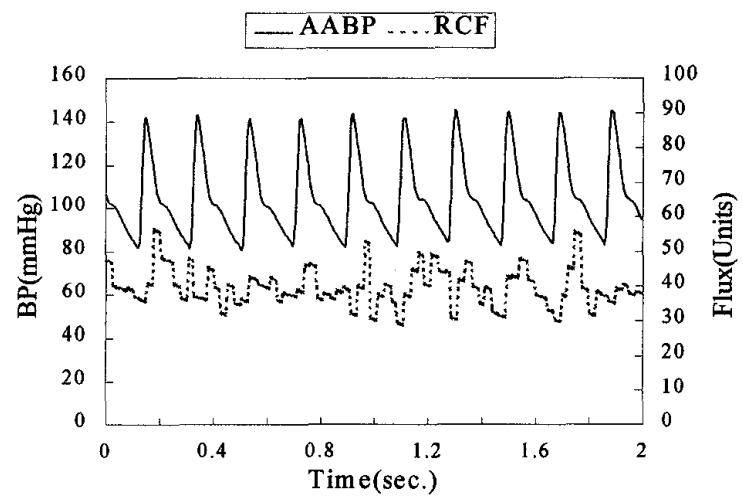

Fig. 1. The abdominal aortic blood pressure (AABP) and the pulsatile renal cortical flux (RCF) measured by a commercial LDF, MBF3.

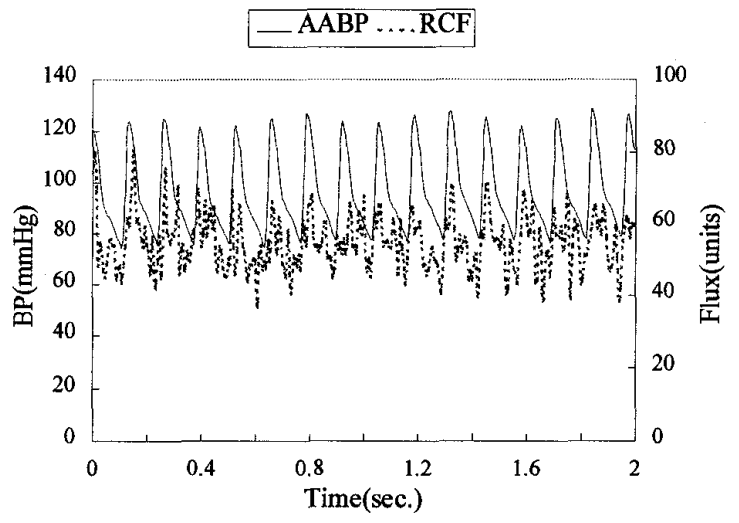

Fig. 2. The abdominal aortic blood pressure (AABP) and the pulsatile renal cortical flux (RCF) measured by a modified fast LDF with $0.021 \mathrm{~s} \mathrm{time} \mathrm{constant.}$

$(\mathrm{CV}=0.015)$; the one using the modified LDF was $102 \pm 2.0$ units $(\mathrm{CV}=0.020)$. Both the $\mathrm{CV}$ were less than $2 \%$ indicates the measurements are stable. Figure 1 demonstrates the flux measured by MBF3, the stair-like shape dues to the DAC output. The flux measured by the modified LDF is shown on figure 2 that clearly exhibites the renal cortical flux is pulsatile.

\section{Discussion}

Alghough the theoretical background of the pulsatile microcirculation has not clearified, some observation had proved the existance of the pulsatile microcirculation in maliman $[1,3,6]$ and in frogs [2] with different kind of tools. LDF is a better tool to observe the microcirculation with less preparation than other means. Thus, a fast LDF provides a new way to observe the pulsatile microcirculation in the rat model.

\section{References}

[1] M. Intaglietta, W.R. Tompkins, and D.R. Richardson, "Velocity Measurement in the Microvasculature of the Cat Omentum by On-line Method," Microvasc. Res., vol. 2, PP. 462-473, 1970.

[2] K. Tyml and A.C. Groom, "Fourier Transform Analysis of Periodic Variations of Red Cell Velocity in Capillaries of Resting Skeletal Muscle in Frog," Microvasc. Res., vol. 10, pp. 9-18, 1980.

[3] J.J. Lee, K. Tyml, A. H. Menkis, R.J. Novick and F.N. Mckenzie, "Evaluation of Pulsatile and Nonpulsatile Flow in Capillaries of Goat Skeletal Muscle Using Intravital Microscopy," Microvasc. Res., vol. 48, pp. 316-327, 1994.

[4] A. P. Shepherd and P.А. Öberg ed., Laser-Doppler Blood Flowmetry., Kluwer Academic Publishers, BOSTON, 1990.

[5] R. Bonner and R. Nossal, "Model for Laser Doppler Measurements of Blood Flow in Tissue," Appl. Opt., vol. 20, pp. 2097-2107, 1981.

[6] M.Y. Jan, T.L. Hsu, Y.Y. Lin Wang and W.K. Wang, "A cyclocorrelogram to analyse the fast Laser-Doppler Flowmetery", Proceeding of World Congress on Medical Physics and Biomedical Engineering, Nice, France, Sep. 1419, p.626, 1997.

M.Y. Jan, T.L. Hsu, H. Hsiu, Y.Y. Lin Wang and W.K. Wang, "Using Fast Laser Doppler Flowmetery with an Averaged Periodogram to Analyze the Microcirculation on the Renal Cortex in Rats," Full paper Sumitted to IEEE Trans. on BME. 